\title{
Design and Research of Automatic Tool Changer System for Multistation Spring Forming Machine
}

\author{
Wei Li $(1),{ }^{1,2}$ Decheng Wang, ${ }^{2}$ and Peng Cheng ${ }^{1}$ \\ ${ }^{1}$ China Productivity Center for Machinery, China Academy of Machinery Science and Technology, 2 Shouti South Road, \\ Haidian District, Beijing, China \\ ${ }^{2}$ China Academy of Machinery Science and Technology, 2 Shouti South Road, Haidian District, Beijing, China
}

Correspondence should be addressed to Wei Li; liweicam@163.com

Received 7 January 2020; Accepted 17 November 2020; Published 1 December 2020

Academic Editor: L. Fortuna

Copyright (c) 2020 Wei Li et al. This is an open access article distributed under the Creative Commons Attribution License, which permits unrestricted use, distribution, and reproduction in any medium, provided the original work is properly cited.

\begin{abstract}
Currently, the spring machine tool is fixed by means of manual clamping and positioning by bolts. The automation of the tool change is low, and the accurate value of the tool position cannot be determined. The automatic tool change system for spring machine was designed by using the standard functional parts of the automatic tool change system of the machining center. It mainly includes the design of the automatic loose clamp device and that of the multispindle automatic tool change arm. After static analysis, the deformation of the automatic tool change arm is $0.153 \mathrm{~mm}$, which satisfies the requirements for usage. Kinematic analysis of the designed automatic tool change arm was carried out. After actual verification, the designed automatic tool change system of spring machine realizes the functions of the tools automatic replacement and positioning of the spring machine, which improves the automation of the spring forming equipment.
\end{abstract}

\section{Introduction}

With the continuous development of manufacturing technology, the automation in the field of machinery continues to improve. Due to the introduction of the automatic tool magazine system, the metal cutting machine tool realizes the functions of automatically storing tools and tool change in the automatic machining process. Various machining processes such as milling, boring, drilling, and tapping can be completed through the control of the CNC system. The change of different tools greatly improves the efficiency of parts processing and shortens the processing time [1-3].

Spring is an important basic mechanical part, and the level of spring manufacturing device is the key to the quality and efficiency of spring production. The current spring forming machine with high automation is the multistation camless spring forming machine. The forming process is that the steel wire is straightened by the straightening mechanism and is sent out from the forming panel mandrel by the wire feeding mechanism. The forming panel is generally equipped with eight linear motion sliding seats, each of which is separately controlled by the servo motor. The spring forming is realized by installing different tools on each sliding seat using multiaxis interpolation control technology.

Currently, spring forming tool is manually mounted on the tool mounting seat on the sliding seat and is positioned and clamped using bolt. Replacement of the forming object requires all the eight tool seats to be removed and replaced with new ones. The tool also needs to be manually replaced when it wears, and the relative position of the tool, the wire, and the mandrel after manual replacement of the tools with different functions and shapes cannot be determined. It requires recommission of the spring forming program, which is very inconvenient. The perfection of the dynamical system has been getting more and more attention [4, 5]. Therefore, an automatic tool change system is designed for the multistation spring forming machine. It can determine the exact relative position of the tool and realize the automatic replacement of the spring tool. Thereby, it provides a basis for further realizing the digital programming technology of the spring and improving the level of manufacturing equipment of the spring parts. 


\section{Design of the Automatic Loose-Clamp Device of Tool}

2.1. Existing Tool Mounting Methods. The existing tool of the spring forming machine is mounted on the tool mounting seat on the linear motion sliding seat, as shown in Figure 1. The tool seat can, respectively, adjust the upper, lower, left and right, and upward and overhead positions of the tool by bolts. In the manual tool change process, the operator needs loosening a few screws, removing the tools, selecting the appropriate tools from the tool magazine, installing the tools, and tightening the screws.

The tool of the machining center has the relevant national standard, so the tools of the machining center produced by different manufacturers can be used universally according to the standard. But, the spring machine tools are still unstandardized. Generally, manufacturers or users customize their own sharpening, so the shape cannot be unified. The tool needs to be adjusted to a suitable position in use by various adjustment bolts on the tool seat to facilitate forming processing. After adjusting a suitable position, the operator needs to constantly adjust the feed of the tool until the formed spring meets the requirements. It will take a lot of time during machine adjustment by manually changing the tools. It seriously reduces the production efficiency of the spring machine. In order to reduce the adjustment time and improve the level of automation of spring machine, the design of automatic tool change system for spring machine is necessary.

2.2. Tool Loose-Clamp Device Structure Design. In order to realize the function of automatic tool change, it is necessary to first realize the automatic loose-clamp function of the tool. At present, this method of using bolts for tightening and positioning is difficult to realize automation, and it cannot be docked with the tool magazine system. In the machining center, the rivet is released by driving the claw under the action of the unclamping cylinder, and it is tightened by driving the claw under the tension of disc spring. The action of the automatic loose-clamp tool holder of the spindle can be realized, and the handle is clamped by the conical surface and the spindle $[6,7]$. Therefore, drawing on the idea of tool loosening and clamping in the machining center, a device for automatically loosening and clamping the tool is designed on the linear slide of the spring machine forming panel using the existing common parts of the standard CNC machine tool. The device is shown in Figures 2 and 3, mainly including parts such as a tool holder, a rivet, a claw, a tool cylinder, a tool cylinder fixture, a cylinder, a connecter of the cylinder and claw, and a cylinder connecting plate. The BT standard is commonly used in the tool holder, rivet, and claw in machining centers [8]. The internal dimensions of BT standard spindle are shown in Figure 4. The tool of the spring machine is rectangular usually, and in this device, the end of the tool can be changed to a cylindrical shape, which can be installed in the tool holder using the ER chuck.
2.3. Loose-Clamp Principle of the Tool. The loosening and clamping actions of the tool is achieved by using the cylinder. When the cylinder action rod pushes the claw to advance, the claw can release the rivet and the $\mathrm{CNC}$ tool holder can be loosened and removed; when the cylinder action rod pulls the claw back, the claw can grasp the rivet to make the conical surface of the tool holder axially positioned and clamped with the conical surface of the tool cylinder. Since general spring machine tools have no rotation action and only move in a straight line, the installation direction of the tool is fixed. Therefore, in addition to the axial positioning, the tool holder also needs to maintain accurate circumferential positioning. The tool holder-matching key with arc shape is designed and mounted on the tool cylinder. When the tool holder is loaded into the tool cylinder, the key groove on the tool holder is automatically aligned along the circular arc surface of the key to achieve circumferential positioning.

The entire tool cylinder is fixed to the linear slide by the fixture and the connecting plate. The cylinder is also fixed to the slide by a connecting plate. Eight forming linear slides are all designed to be installed with this device to achieve the automatic loose-clamp action of the tools on the eight stations. They are shown in Figure 5. The tool can be directly used for forming processing after it is replaced.

\section{Automatic Tool Change Arm Design}

In order to realize automatic tool change, it is necessary to design an automatic tool change arm for the spring machine. The common tool change in the machining center is achieved through the ATC device. In the working process, it is mainly responsible for the exchange of the tools on the tool magazine with the tools on the machine tool spindle. Through a series of rotation and linear motion, it can achieve the efficient and accurate exchange of the tool required for the next process in the tool magazine with the tool used in the previous process in the machine spindle $[9,10]$.

There is a very large difference of the tool change process between the multistation spring machine and the machining center. In the machining center, it is generally one-to-one tool change between tool magazine with the single spindle. In the spring machine, it is one-to-eight tool change between tool magazine with the spindles of the eight stations which are distributed on the panel at a 45 degree circumference. Therefore, the tool change arm used in the machining center cannot be directly used on the spring forming machine. It is necessary to design an automatic tool change arm that is suitable for the characteristics of the spring machine.

\subsection{Structure Design of the Automatic Tool Change Arm.} The structure of the spring machine tool change mechanism is shown in Figure 6. It adopts the form of single-arm and single-handle. It includes a servo motor, a reducer, and four cylinders, which, respectively, realizes two rotary motions, two linear motions, and opening and closing motions of the clamping jaw. In this way, it provides the movement of four degrees of freedom for the clamping jaw of the tool holder 


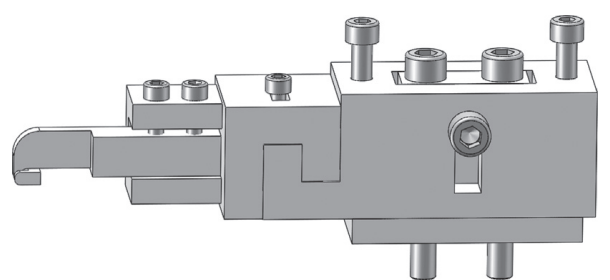

FIGURE 1: Schematic diagram of tool installation adjustment seat on the spring forming machine.

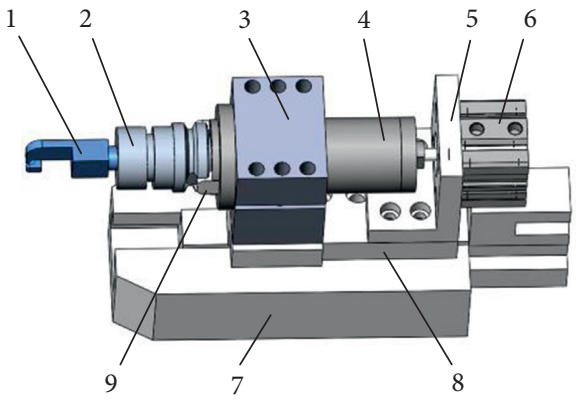

$$
\begin{array}{lc}
\text { 1 Spring forming tool } & \text { 6 Cylinder } \\
\text { 2 BT CNC tool holder } & \text { 7 Spring machine } \\
\text { 3 Tool cylinder fixture } & \text { linear slide } \\
\text { 4 Tool cylinder } & \text { 8 Connecting plate } \\
\text { 5 Cylinder connecter } & \text { 9 Tool holder arc key }
\end{array}
$$

FIGURE 2: Schematic diagram of the automatic loose-clamp device.

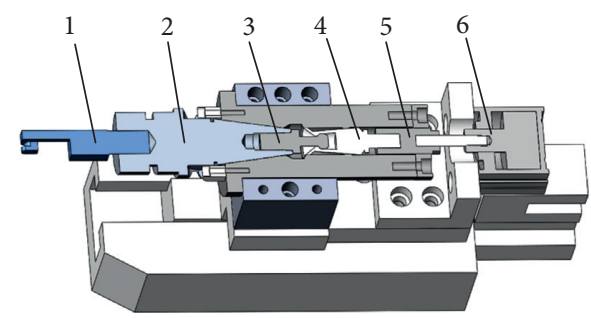

$$
\begin{aligned}
& 1 \text { Spring forming tool } \\
& 2 \text { BT CNC tool holder } \\
& 3 \text { Tool holder rivet } \\
& 4 \text { Claw } \\
& 5 \text { Connecting piece of the cylinder and } \\
& \text { claw 6-cylinder }
\end{aligned}
$$

FIgURE 3: Schematic diagram of the cross-section of the automatic loose-clamp device.

and meets the working requirements of the tool change of the spring machine. The function of the rotary cylinder is to rotate the whole tool change arm between the tool magazine and the spring machine. The function of the motor 6 is to make the angle of the tool change arm coincide with the angle of the slide of the spring machine forming plate from which the tool will be removed or mounted. The cylinder 2 realizes the opening and closing of the clamping jaw, the cylinder 3 ensures that the tool holder does not collide with other parts during the movement process after the tool holder is removed, and the cylinder 4 completes the motions of tool uploading and loading.
3.2. Tool Change Workflow of the Automatic Tool Change Arm. According to the operating principle of the designed spring machine automatic tool change arm, the working process of tool change is divided into the loading process and the unloading process.

The loading process on the spring machine: positioning the tool change arm to the position of the tool magazine (motor 5, cylinder 7), clamping jaw release (cylinder 2), falling down of the tool case with the tool of the tool magazine, moving clamping jaw in place (cylinders 3,4), closing of the clamping jaw (cylinder 2), tool change arm pulling the tool (cylinder 4), the clamping jaw moving back 


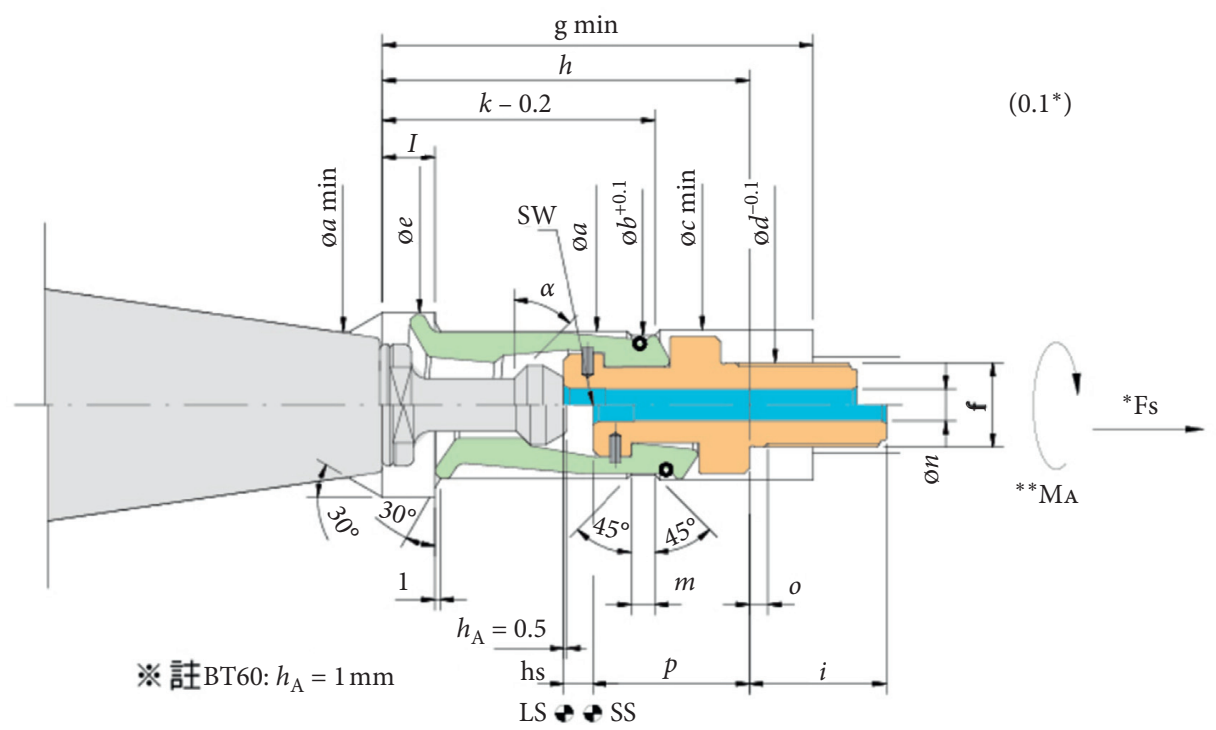

FIgURE 4: Internal structure size of spindle using BT-standard.

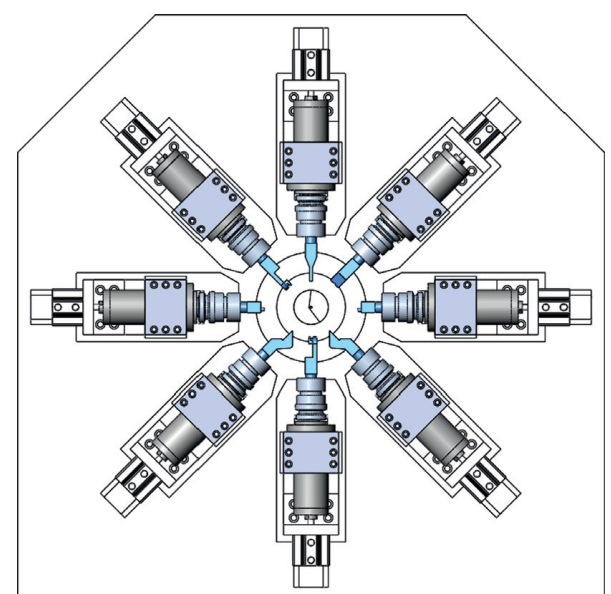

FIGURE 5: Spring formed panel with the automatic loose-clamp function of tool.

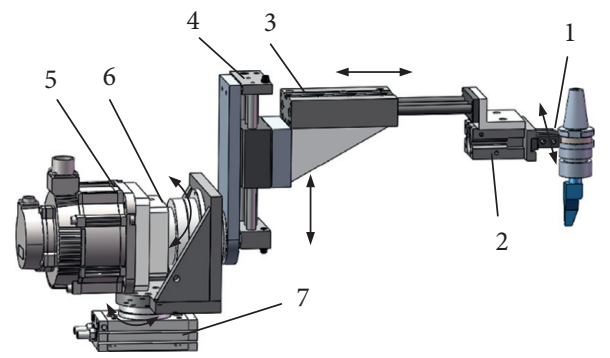

$$
\begin{array}{ll}
1 \text { Pneumatic clamping jaw } & 5 \text { Servo motor } \\
2 \text { Clamping jaw cylinder } & 6 \text { Reducer } \\
3 \text { Linear three-rod cylinder } & 7 \text { Rotary cylinder } \\
4 \text { Linear rodless cylinder } &
\end{array}
$$

Figure 6: Structure of the automatic tool change arm.

(cylinder 3), positioning the tool change arm to the angle of the spring machine for tool loading (motor 5, cylinder 7), tool inserting (cylinders 3,4 ), tightening the tool holder of the automatic loose clamp device, clamping jaw release (cylinder 2), and the tool change arm returning to the initial position (cylinders 3,4 , motor 5 ). 
The unloading process on the spring machine: positioning the tool change arm to the angle of the spring machine for tool unloading (motor 5, cylinder 7), clamping jaw release (cylinder 2), moving clamping jaw in place (cylinders 3, 4), closing of the clamping jaw (cylinder 2), tool loosening of the automatic loose-clamp device, tool change arm pulling the tool (cylinder 4), the clamping jaw moving back (cylinder 3), positioning the tool change arm to the position of the tool magazine (motor 5, cylinder 7), falling down of the empty tool case of tool magazine, tool inserting (cylinders 3, 4), clamping jaw release (cylinder 2), and the tool change arm returning to the initial position (cylinders 3, 4, motor 5).

3.3. Static Analysis of the Automatic Tool Change Arm. In order to verify whether that the designed automatic tool change arm meets the requirements for use, the structural static analysis of the structure is conducted using ANSYS WORKBENCH finite element software [11, 12]. The 3D model of the designed automatic tool change arm is imported into ANSYS WORKBENCH. The automatically divided grid is adopted, and there are altogether 392,883 nodes and 226,738 units. In the case of clamping the tool holder, the maximum deformation of the structure is about $0.153 \mathrm{~mm}$ under the effect of its own weight. The deformation result is shown in Figure 7. It indicates that the deformation of the tool change arm structure is small during the using process, which will not affect the positioning accuracy and fully meet the requirements of use.

3.4. Kinematics Analysis of the Automatic Tool Change Arm. The D-H method is a matrix method proposed by Denavit and Hartenberg to model the robot linkages and joints and to describe the translation and rotation relationship of adjacent members, which has become the standard method for robot kinematics modeling [13-17]. It establishes an additional coordinate system for the members at each joint and represents the relationship between the coordinate systems by a $4 \times 4$ homogeneous transformation matrix.

The transformation matrix between joint $n-1$ and joint $n$ is

$$
{ }^{n-1} T_{n}=A_{n}=\left[\begin{array}{cccc}
C \theta_{n} & -S \theta_{n} C \partial_{n} & S \theta_{n} S \partial_{n} & a_{n} C \theta_{n} \\
S \theta_{n} & C \theta_{n} C \partial_{n} & -C \theta_{n} S \partial_{n} & a_{n} S \theta_{n} \\
0 & S \partial_{n} & C \partial_{n} & d_{n} \\
0 & 0 & 0 & 1
\end{array}\right],
$$

where $\quad S \theta_{n}=\sin \theta_{n}, \quad C \theta_{n}=\cos \theta_{n}, \quad S \partial_{n}=\sin \partial_{n}, \quad$ and $C \partial_{n}=\cos \partial_{n}$.

The self-designed tool change arm consists of the joints of two rotational degrees of freedom and joints of two translational degrees of freedom. The coordinate system at each joint of the tool change arm is established according to the D-H method, as shown in Figure 8. The D-H parameters of each coordinate system conversion are shown in Table 1.

$\theta_{1}$ is determined by the operating angle of the rotating cylinder; $\theta_{2}$ is determined by the rotation angle of the motor; $d_{2}$ is the linear distance from the center line of the rotating cylinder to the center line of the rodless cylinder, which is a fixed value; $d_{3}$ is determined by the stroke of the rodless cylinder; $d_{4}$ is determined by the stroke of the three-rod cylinder. The characteristic of cylinder as the actuating action element is that, generally, it can only stay at both ends within the stroke range, but it can meet the requirements and save the cost in this mechanism. The value range of each parameter is shown in Table 2.

By substituting the $\mathrm{D}-\mathrm{H}$ parameters into formula 1 , the coordinate transformation matrix between the connecting rods can be obtained as

$$
\begin{aligned}
& A_{1}=\left[\begin{array}{cccc}
C \theta_{1} & 0 & -S \theta_{1} & 0 \\
S \theta_{1} & 0 & C \theta_{1} & 0 \\
0 & -1 & 0 & 0 \\
0 & 0 & 0 & 1
\end{array}\right], \\
& A_{2}=\left[\begin{array}{cccc}
C \theta_{2} & 0 & S \theta_{2} & 0 \\
S \theta_{2} & 0 & -C \theta_{2} & 0 \\
0 & 1 & 0 & d_{2} \\
0 & 0 & 0 & 1
\end{array}\right], \\
& A_{3}=\left[\begin{array}{cccc}
1 & 0 & 0 & 0 \\
0 & 0 & 1 & 0 \\
0 & -1 & 0 & d_{3} \\
0 & 0 & 0 & 1
\end{array}\right], \\
& A_{4}=\left[\begin{array}{llll}
0 & -1 & 0 & 0 \\
1 & 0 & 0 & 0 \\
0 & 0 & 1 & d_{4} \\
0 & 0 & 0 & 1
\end{array}\right] .
\end{aligned}
$$

Then, the position and orientation transformation matrix of the coordinate system of the center of clamping jaw at the end of the entire tool change the mechanism relative to the base coordinate system, i.e., the kinematic equation of the entire tool change mechanism is 


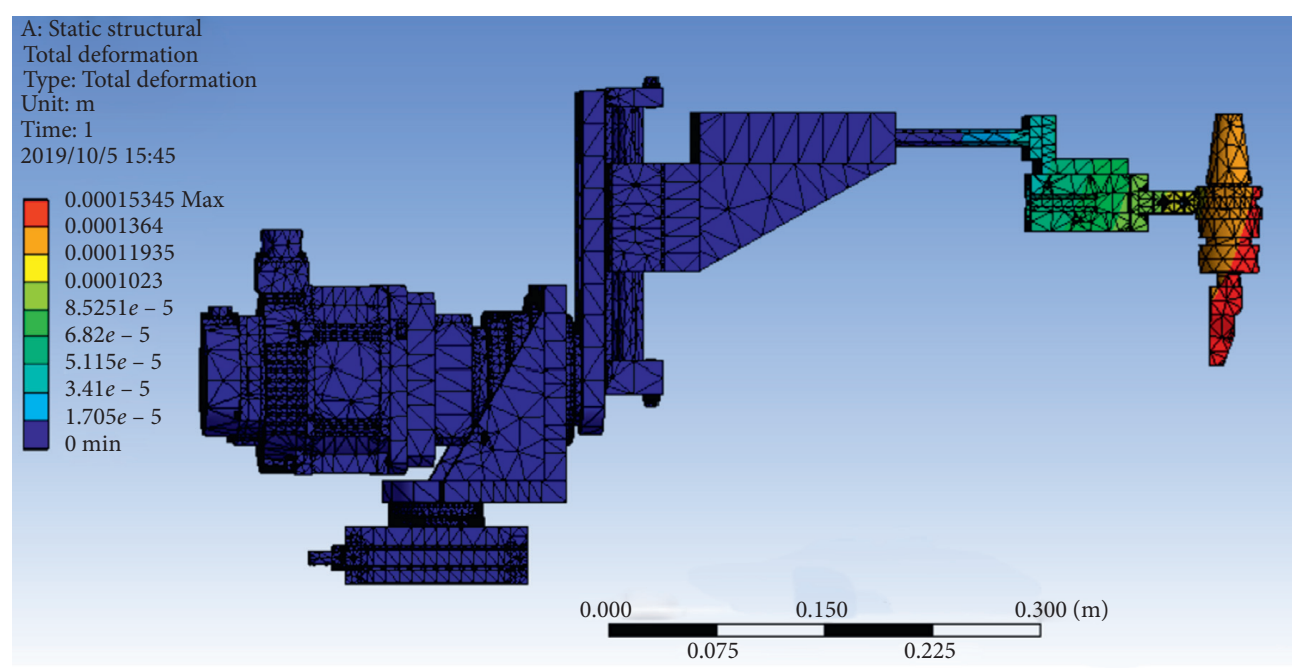

FIGURE 7: Static mechanical finite element analysis of the structure of the tool change arm.

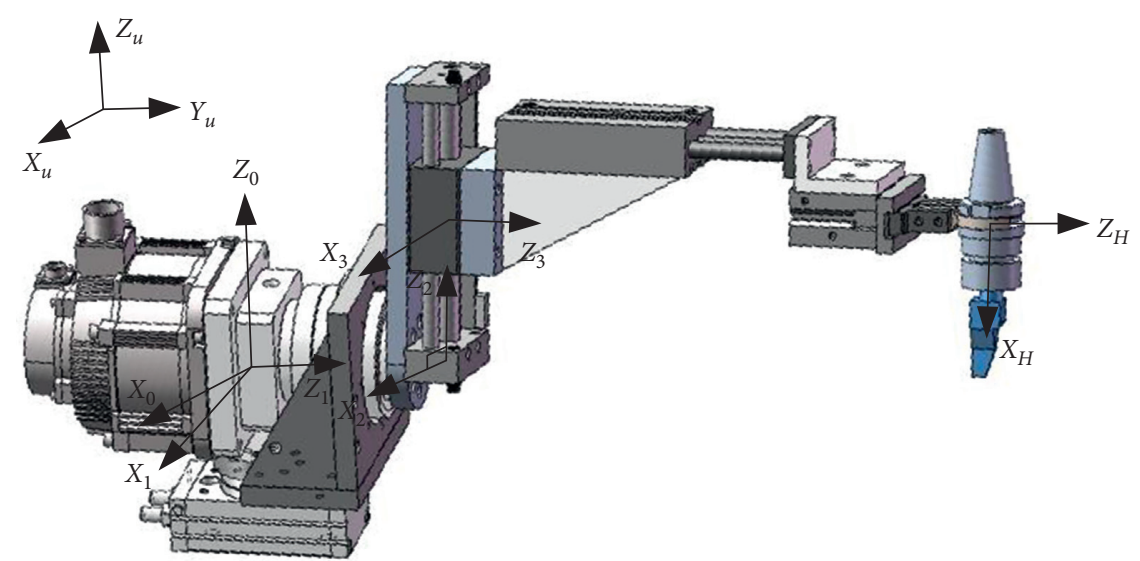

FIgURE 8: Schematic diagram of multiaxis coordinate system of tool change arm kinematics.

TABle 1: D-H parameter table.

\begin{tabular}{lcccc}
\hline$\#$ & $\theta$ & $\mathrm{D}$ & $\mathrm{a}$ & \\
\hline $0-1$ & $\theta_{1}$ & 0 & 0 & A \\
$1-2$ & $\theta_{2}$ & $d_{2}$ & 0 & -90 \\
$2-3$ & 0 & $d_{3}$ & 0 & 90 \\
$3-\mathrm{H}$ & 90 & $d_{4}$ & 0 & -90 \\
\hline
\end{tabular}

TABle 2: The value range of $\mathrm{D}-\mathrm{H}$ parameters.

\begin{tabular}{lccccc}
\hline Parameter & $\theta_{1}\left(^{\circ}\right)$ & $\theta_{2}\left(^{\circ}\right)$ & $d_{2}(\mathrm{~mm})$ & $d_{3}(\mathrm{~mm})$ & 60.5 \\
\hline Lower limit of value & 0 & 0 & 115 & $d_{4}(\mathrm{~mm})$ \\
Upper limit of value & 90 & 360 & 115 & 150.5 & 350 \\
\hline
\end{tabular}




$$
\begin{aligned}
{ }^{0} T_{H} & =A_{1} \times A_{2} \times A_{3} \times A_{4}, \\
& =\left[\begin{array}{cccc}
-C \theta_{1} S \theta_{2} & -C \theta_{1} C \theta_{2} & -S \theta_{1} & d_{3} C \theta_{1} S \theta_{2}-d_{4} S \theta_{1}-d_{2} S \theta_{1} \\
-S \theta_{1} S \theta_{2} & -S \theta_{1} C \theta_{2} & C \theta_{1} & d_{2} C \theta_{1}+d_{4} C \theta_{2}+d_{3} S \theta_{1} S \theta_{2} \\
-C \theta_{2} & S \theta_{2} & 0 & d_{3} C \theta_{2} \\
0 & 0 & 0
\end{array}\right], \\
{\left[\begin{array}{ccc}
n_{x} & o_{x} & a_{x} \\
n_{y} & o_{y} & a_{y} \\
n_{z} & o_{z} & a_{z}
\end{array}\right]=} & {\left[\begin{array}{ccc}
-C \theta_{1} S \theta_{2} & -C \theta_{1} C \theta_{2} & -S \theta_{1} \\
-S \theta_{1} S \theta_{2} & -S \theta_{1} C \theta_{2} & C \theta_{1} \\
-C \theta_{2} & S \theta_{2} & 0
\end{array}\right], } \\
{\left[\begin{array}{c}
p_{x} \\
p_{y} \\
p_{z}
\end{array}\right]=} & {\left[\begin{array}{ll}
d_{3} C \theta_{1} S \theta_{2}-d_{4} S \theta_{1}-d_{2} S \theta_{1} \\
d_{2} C \theta_{1}+d_{4} C \theta_{2}+d_{3} S \theta_{1} S \theta_{2} \\
d_{3} C \theta_{2}
\end{array}\right], }
\end{aligned}
$$

where $\left[\begin{array}{lll}n_{x} & o_{x} & a_{x} \\ n_{y} & o_{y} & a_{y} \\ n_{z} & o_{z} & a_{z}\end{array}\right]$ is the orientation transformation matrix of the coordinate system of the center of clamping jaw at the end of the entire tool change mechanism relative to the base coordinate system and $\left[\begin{array}{l}p_{x} \\ p_{y} \\ p_{z}\end{array}\right]$ is the position transformation matrix of the coordinate system of the center of clamping jaw at the end of the entire tool change mechanism relative to the base coordinate system.

Through the above kinematic equation, if $\theta_{1}, \theta_{2}, d_{2}, d_{3}$, and $d_{4}$ are known, the position and orientation of the clamping jaw can be obtained by substituting them into the above matrix, i.e., the positive kinematic solution of the tool change arm; if the desired position and orientation value of the clamping jaw are known, the values of $\theta_{1}, \theta_{2}, d_{2}, d_{3}$, and $d_{4}$ can be solved through the above matrix, i.e., the motion values of the joints, which is the inverse kinematics solution of the tool change arm. Through the results of kinematic analysis, related guidance parameters can be provided for the control system of the tool change arm.

\section{Selection of Tool Magazine Types}

The common types of tool magazines for machining center include circular type [18], bamboo-hat type, and chain type. Different types of tool magazines have different characteristics and usage scenarios. Among them, the circular and chain tool magazines generally use automatic tool change mechanism for tool exchange. Since spring machine is in the form of multispindle tool change, it is suitable to use automatic tool change mechanism ATC for tool exchange. Therefore, circular tool magazine can be equipped generally, and chain tool magazine can be used when the number of stored tools is large. The circular tool magazine just needs the functions of tool selecting and tool case turning, and the function of tool change is realized by the self-designed multispindle automatic tool change arm. The structure of the spring machine equipped with an automatic tool change system using the circular tool magazine is shown in Figure 9.

\section{Physical Test Verification}

In order to verify the functions of the designed tool automatic clamping device and automatic tool changing arm, a physical model is built in this paper. It is shown in Figure 10. The open control system of IPC + PMAC motion control card is used to build the control system of automatic tool change system [19]. The system controls the actions of 5 cylinder reversing valves and 1 servo motor. IPC, as the upper computer, completes human-computer interaction and management functions. And PMAC, as the lower computer, completes motion control and logic control functions. A special control system for automatic tool change mechanism of multistation spring machine is developed on the Windows platform based on C\# language. It realizes the functions of automatic tool change mechanism, such as automatic loosening and clamping tool, automatic unloading and loading tool control, and tool information management.

Through actual operation, the function of automatic loosening and clamping of the tool on spring forming machine and the function of the automatic tool change between the magazine and the spring machine are verified to be valid.

\section{Discussion}

(1) The mechanism designed in this paper can realize the computer-controlled tool change process, reduce the manual operation, improve the level of automation, and provide the foundation for the future unmanned intelligent manufacturing.

(2) After actual testing, the fastest tool change of one station can be achieved in about 1 minute by automatic tool change. And, the tool change time manually depends on the operator's proficiency and operating speed. In terms of tool change time alone, the level of improvement may be limited.

(3) The position of the tool after the automatic tool change is determined. According to the shape of the spring to be formed, it is easy to infer the appropriate 


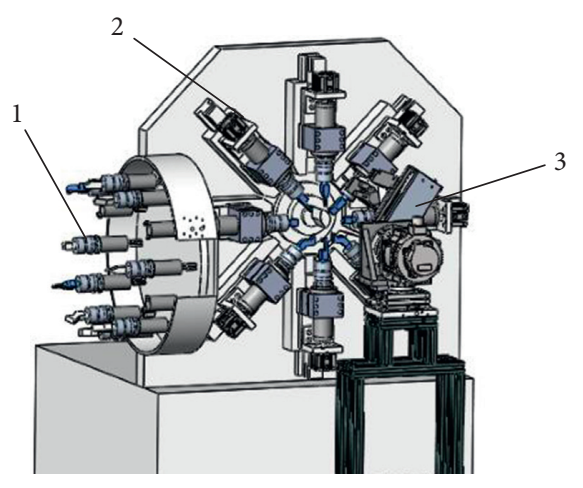

1 Circular tool magazine

2 Spring forming panel

3 Automatic tool change arm

Figure 9: Schematic diagram of the spring machine with the automatic tool change system.

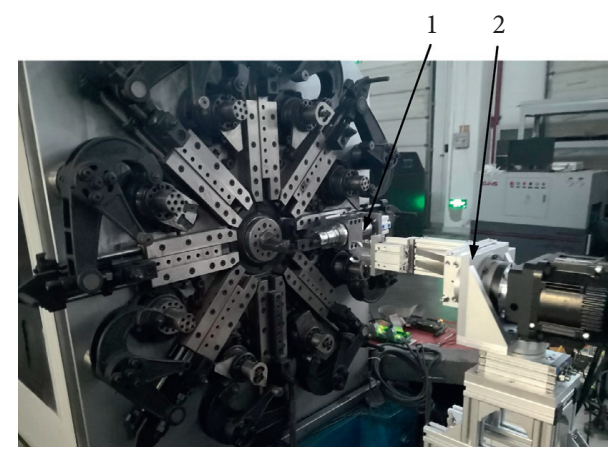

1 Automatic loose-clamp device

2 Automatic tool change arm

Figure 10: Physical prototype model.

feed of the tool. But, after manual tool change, the tool position is difficult to determine; it needs repeated tests to determine the appropriate tool feed. Usually, the manual debugging spring program needs at least half an hour. From this point of view, the design of automatic tool change system greatly reduces the adjustment time of the program. When the machining object of spring forming machine changes, it can be adjusted quickly. Compared with the method of manual tool change, it improves the proportion of actual processing time. It can give full play to the advantages of multistation spring machine in multivarieties and small batch production.

(4) By using the automatic tool change system, the tool standardization of the spring machine can be promoted, which provides the basic conditions for the development of CAM technology in the spring forming process.

(5) On the basis of automatic tool change of the spring machine, the computer reasoning of tool selection and layout based on experience knowledge can be further realized, so as to get rid of the dependence on the experience of operators.

\section{Conclusion}

(1) Through the automatic tool change system designed for the multistation spring machine, the functions of automatic clamping, automatic replacement, and automatic storage of the spring machine tool are achieved by using the standard parts commonly used in the tool magazine system of machining center, and the reliability is ensured.

(2) The device for automatically loosening and clamping the tool in circumference distribution of the spring machine forming panel is designed. The circular tool magazine is selected, and the automatic tool change arm of the tool magazine and multispindle tool change is designed. The operating principle of each part is illustrated.

(3) The statics analysis is conducted for the mechanics of the structure of the tool change arm. The results showed this structure meets the requirements of use. And, kinematics analysis is also conducted for the mechanics of the structure of the tool change arm. The kinematics equations for these mechanisms are obtained.

(4) The designed structures are verified on the actual physical prototype. The result shows that the structure of the design is reasonable and the function of the design can be realized.

(5) The entire automatic tool change system designed can be applied to the multistation spring machine, which is important for improving the spring manufacturing device, promoting the standardization process of spring machine tools, and realizing the digitization of formation of spring.

\section{Data Availability}

The data used to support the findings of this study are available from the corresponding author upon request.

\section{Conflicts of Interest}

The authors declare that they have no conflicts of interest.

\section{Acknowledgments}

The work described in this paper was fully supported by the National Science and Technology Major Project of China (2018ZX04020001).

\section{References}

[1] L. Hu, Y. Liu, C. Peng, W. Tang, R. Tang, and A. Tiwari, "Minimising the energy consumption of tool change and tool path of machining by sequencing the features," Energy, vol. 147, pp. 390-402, 2018.

[2] E. Niemi, "Optimal tool magazine operation. part 2: rotating magazines with buffered tool change," The International Journal of Advanced Manufacturing Technology, vol. 24, no. 78, pp. 567-572, 2004.

[3] A. Baykasoğlu and F. B. Ozsoydan, "Minimizing tool switching and indexing times with tool duplications in automatic 
machines," The International Journal of Advanced Manufacturing Technology, vol. 89, no. 5-8, pp. 1775-1789, 2017.

[4] M. Bucolo, A. Buscarino, C. Famoso, L. Fortuna, and M. Frasca, "Control of imperfect dynamical systems," Nonlinear Dynamics, vol. 98, no. 4, pp. 2989-2999, 2019.

[5] A. Buscarino, C. Famoso, L. Fortuna et al., "Passive and active vibrations allow self-organization in large-scale electromechanical systems," International Journal of Bifurcation and Chaos, vol. 26, no. 7, 2016.

[6] H. Shen, H. Dong, D. Wang et al., "A novel impact load model for tool-changer mechanism of spindle system in machine tool," The International Journal of Advanced Manufacturing Technology, vol. 94, no. 1-4, pp. 1477-1490, 2018.

[7] H. Tian, Z. Yang, G. Li, and C. Chen, "Study on failure warning of tool magazine and automatic tool changer based on tool-pulling force," Journal of Mechanical Science and Technology, vol. 33, no. 9, pp. 4371-4381, 2019.

[8] Technical Committee on Industrial Automation, JIS B6339-1998 Machining Centers-Tool Shanks and Retention Knobs, Japanese Standards Association, Japan, 1998.

[9] X. Lu, Z. Jia, S. Gao, and P. Han, "Failure mode effects and criticality analysis (FMECA) of circular tool magazine and ATC," Journal of Failure Analysis and Prevention, vol. 13, no. 2, pp. 207-216, 2013.

[10] J.-W. Kim, S. Lee, T. Seo, and J. Kim, "A new non-servo motor type automatic tool changing mechanism based on rotational transmission with dual four-bar linkages," Meccanica, vol. 53, no. 9, pp. 2447-2459, 2018.

[11] T. J. R. Hughes, The Finite Element Method: Linear Static and Dynamic Finite Element Analysis, Courier Corporation, North Chelmsford, MC, USA, 2012.

[12] E. Madenci and I. Guven, The Finite Element Method and Applications in Engineering Using ANSYS ${ }^{\circledR}$, Springer, New York, NY, USA, 2015.

[13] M. B. Vaghela, V. J. Savsani, and S. B. Jadeja, "Design and kinematic analysis of an automatic tool changing mechanism used in VMC," in Proceedings of International Conference on Advances in Tribology and Engineering Systems, pp. 269-283, Springer, New Delhi, January 2014.

[14] C. Berceanu, D. Tarnita, S. Dumitru et al., "Forward and inverse kinematics calculation for an anthropomorphic robotic finger," New Trends in Mechanism Science, pp. 335-342, Springer, Dordrecht, 2010.

[15] Y. Lu, Z. Dai, N. Ye, and P. Wang, "Kinematics/statics analysis of a novel serial-parallel robotic arm with hand," Journal of Mechanical Science and Technology, vol. 29, no. 10, pp. 4407-4416, 2015.

[16] S. S. Roy and D. K. Pratihar, "Kinematics, dynamics and power consumption analyses for turning motion of a six-legged robot," Journal of Intelligent \& Robotic Systems, vol. 74, no. 3-4, pp. 663-688, 2014.

[17] B. He, L. Han, Y. Wang et al., "Kinematics analysis and numerical simulation of a manipulator based on virtual prototyping," The International Journal of Advanced Manufacturing Technology, vol. 71, no. 5-8, pp. 943-963, 2014.

[18] X. H. Lu, P. Z. Han, W. Y. Wu et al., "Reliability evaluation of circular tool magazine and automatic tool changer," Advanced Materials Research, Trans Tech Publications, vol. 630, pp. 245-248, 2013.

[19] X.-m. Xu, Y. Li, J.-h. Sun, and S.-g. Wang, "Research and development of open CNC system based on PC and motion controller," Procedia Engineering, vol. 29, pp. 1845-1850, 2012. 